\title{
International consensus classification of hippocampal sclerosis and etiologic diversity in children with temporal lobectomy
}

\author{
Ceren Günbey ${ }^{\text {a,1 }}$, Figen Söylemezoğlu ${ }^{\text {b,1 }}$, Burçak Bilginer ${ }^{c}$, Kader Karlı Oğuz d ${ }^{\text {, Nejat Akalan }}{ }^{\text {c,2 }}$, Meral Topçu ${ }^{\text {a,3 }}$, \\ Güzide Turanlı ${ }^{\mathrm{a}, 3}$, Dilek Yalnızoğlu ${ }^{\mathrm{a}, *}$
}

a Department of Pediatric Neurology, Hacettepe University Faculty of Medicine, Ankara, Turkey

${ }^{\mathrm{b}}$ Department of Pathology, Hacettepe University Faculty of Medicine, Ankara, Turkey

c Department of Neurosurgery, Hacettepe University Faculty of Medicine, Ankara, Turkey

d Department of Radiology, Hacettepe University Faculty of Medicine, Ankara, Turkey

\section{A R T I C L E I N F O}

\section{Article history:}

Received 8 April 2020

Revised 23 July 2020

Accepted 27 July 2020

Available online 1 September 2020

\section{Keywords:}

Epilepsy surgery

Children

Hippocampal sclerosis

International consensus classification

Long-term seizure outcome

\begin{abstract}
A B S T R A C T
Introduction: The distribution of hippocampal sclerosis (HS) subtypes, according to the classification of the International League Against Epilepsy (ILAE), has been reported mainly in adult patients. We aimed to review the pathological findings in children who had anterior temporal lobectomy accompanied with amygdalohippocampectomy, in view of the current classification, and evaluate postsurgical outcome with respect to HS subtypes in childhood. Methods: Seventy children who underwent temporal resections for treatment of medically refractory epilepsy, with a minimum follow-up of 2 years, were included; the surgical hippocampus specimens were re-evaluated under the HS ILAE classification.

Results: Neuropathological evaluations revealed HS type 1 in 38 patients (54.3\%), HS type 2 in 2 (2.8\%), HS type 3 in 21 patients (30\%), and no HS in 9 patients (12.9\%). Of 70 patients, 23 (32.9\%) had dual pathology, and the most common pattern was HS type 3 with low-grade epilepsy-associated brain tumors (LEAT). The distribution of HS types with respect to age revealed that HS type 3 and no HS subgroups had significantly more patients younger than 12 years, compared with those of HS type 1 ( $90.5 \%, 77.8 \%$ vs $47.4 \%$, respectively). History of febrile seizures was higher in HS type 1. Prolonged/recurrent febrile seizures were most common in patients 12 years and older, whereas LEAT was the most common etiology in patients under 12 years of age $(p<0.001)$. Patients with HS type 1 had longer duration of epilepsy and an older age at the time of surgery compared with patients with HS type 3 and no HS (p: 0.031, p: 0.007). At final visit, $74.3 \%$ of the patients were seizure-free. Seizure outcome showed no significant difference between pathological subtypes.

Conclusions: Our study presents the distribution of HS ILAE subtypes in an exclusively pediatric series along with long-term seizure outcome. The study reveals that the leading pathological HS subgroup in children is HS type 1, similar with adult series. Hippocampal sclerosis type 2 is significantly less in children compared with adults; however, HS type 3 emerges as the second most predominant group because of dual pathology, particularly LEAT. Further studies are required regarding clinicopathological features of isolated HS in pediatric cohort. Seizure-free outcome was favorable and similar in all HS types in children. The proportion of HS types may be better defined in pediatric patients with temporal resections, as the current HS ILAE classification becomes more widely used, and may help reveal the surgical and cognitive outcome with respect to HS types.
\end{abstract}

(c) 2020 Elsevier Inc. All rights reserved.

\footnotetext{
* Corresponding author at: Hacettepe University Faculty of Medicine, Department of Pediatric Neurology, Ankara, Turkey.

E-mail address: dileky@hacettepe.edu.tr (D. Yalnızoğlu).

${ }^{1} \mathrm{CG}$ and $\mathrm{FS}$ contributed equally to this study.

${ }^{2} \mathrm{NA}$ is currently at Istanbul Medipol University.

${ }^{3} \mathrm{MT}$ and GT are currently retired from Hacettepe University.
}

\section{Introduction}

Epilepsy surgery has shown efficacy in both children and adults with refractory temporal lobe epilepsy (TLE) [1]. While hippocampal sclerosis (HS) is the most common pathological substrate in adults undergoing temporal lobe surgery, children have a different pathological profile including low-grade epilepsy-associated brain tumors (LEAT) and focal cortical dysplasia (FCD) [2-5]. Hippocampal sclerosis may be associated with other developmental or acquired lesions in the temporal lobe more in children than adults [3,4]. In 2013, the International 
League Against Epilepsy (ILAE) Commission on Diagnostic Methods published a task force report on the International Consensus Classification of HS in TLE [6]. We reviewed the pathological findings and longterm seizure outcome in pediatric patients who underwent epilepsy surgery involving temporal resections in view of the current classification.

\section{Methods}

\subsection{Study population}

This study was approved by the Institutional Review Board of Hacettepe University Faculty of Medicine.

A total of 236 children underwent epilepsy surgery for treatment of medically refractory epilepsy at Hacettepe University between June 1994 and January 2015. Among them, 125 patients had temporal resections; 81 patients who had anterior temporal lobectomy accompanied with amygdalohippocampectomy $(\mathrm{AH})$ were evaluated according to the International Consensus Classification of HS in TLE. Eleven of 81 specimens were excluded (one with tumor totally infiltrating hippocampus, two with tuberous sclerosis, six with technical problems, and two were not available for review). All tumors included in this study were within ipsilateral anterior temporal lobectomy area. Temporal lobe resections of patients with malignant brain tumors with or without epilepsy were not classified as epilepsy surgery and were excluded.

All cases were discussed prior to surgery in a multidisciplinary epilepsy management conference that included pediatric and adult neurologists/epileptologists, neurosurgeons specialized in pediatric neurosurgery and epilepsy surgery, neuroradiologists, and nuclear medicine physicians.

Information was collected from the medical records regarding patient demographics, medical and surgical history, and seizure history. All patients underwent presurgical evaluation that included routine electroencephalogram (EEG) and/or video-electroencephalographic monitoring, high-resolution $1.5 \mathrm{~T}$ or $3 \mathrm{~T}$ magnetic resonance imaging (MRI). All structural MRI studies applied a dedicated protocol for epilepsy imaging (Sagittal and axial T1-weighted imaging (WI)) (TR/TE; 550-600/15-20 ms), axial FLAIR (TR/TE/TI; 8500-11,000/90-110/ 1900-2100 ms), axial T2WI (TR/TE; 3800-4500/80-100 ms), 3D T1weighted MPRAGE series (TR/TE/TI; 1910/3,5/1100 ms, flip angle:15 ${ }^{\circ}$ ), coronal T2 WI (3750-4500/85-100 ms, slice thickness of $3 \mathrm{~mm}$ ), inversion-recovery (IR) (TR/TE/TI; 6000-6400/75-80/350 ms) obtained perpendicular to hippocampi. Hippocampal sclerosis was defined by atrophy, increased T2 hyperintensity, and loss of internal structure of the hippocampi.

Selected patients underwent functional MRI, fluoro-deoxy glucose positron emission tomography combined with computed tomography (FDG-PET/CT), and/or ictal-interictal single-photon emission computed tomography (SPECT). Neuropsychological evaluation was performed in majority of patients.

The extent of neocortical resection varied depending on the side of surgery, dimension of lesion, and the patient's anatomy. Neocortical resection in anterior temporal lobectomy was usually extended from the tip of temporal pole to nearly $3.5-4 \mathrm{~cm}$ on the dominant and $4-5 \mathrm{~cm}$ on the nondominant hemisphere; it was usually limited by anatomical structures. The hippocampus was fully dissected subpially from the underlying pia and resected at its upward turn behind the quadrigeminal plate.

The postoperative data were obtained from medical records, interviews during the postoperative outpatient visits, or by telephone interviews. All patients had more than 2 years of follow-up after epilepsy surgery. First postsurgical follow-up within 6 months and last followup visit were noted. Surgical outcome was graded according to Engel classification [7].

The neuropathologic specimens were reviewed and classified according to the current HS ILAE classification system by the institutional neuropathologist (FS), who has had 20 years of experience in the field and was blinded to the clinical data, MRI findings, and surgical outcomes [6].

\subsection{Tissue preparation}

Hippocampal and temporal lobectomy specimens were anatomically orientated and cut into 3-5 mm parallel slices according to the anatomic orientation and coded accordingly. All tissues were fixed with formalin overnight, and paraffin-embedded tissue specimens were cut with a rotatory microtome at 4-5 $\mu \mathrm{m}$ thickness. The sections were stained for recommended histochemical (H\&E, cresyl violet) and immunohistochemical [NeuN, phosphorylated and nonphosphorylated neurofilament protein, synaptophysin, glial fibrillary acidic protein (GFAP)] stains.

\subsection{The clinicopathological classification system for hippocampal sclerosis}

The International Consensus Classification of HS in TLE by ILAE Commission on Diagnostic Methods was used to identify the HS types in the specimens [6]. Hippocampal sclerosis ILAE type 1 shows severe neuronal cell loss and gliosis predominantly in CA1 and CA4 regions, HS ILAE type 2 demonstrates predominant neuronal cell loss and gliosis in CA1 region, and HS ILAE type 3 shows predominant neuronal cell loss and gliosis in CA4 region. No HS demonstrates no neuronal loss but gliosis. Even though there is no international consensus on the definition of dual pathology, HS with second principal pathology, i.e., tumor, vascular malformation, glial scar, limbic/Rasmussen encephalitis, or cortical malformation (including FCD type IIa/IIb), was defined as "dual" pathology [8]. Architectural abnormalities in the temporal lobe associated with HS were referred as FCD Type IIla and was not classified as dual pathology [8].

\subsection{Statistical analysis}

Statistical analysis was performed using the Statistical Package for the Social Sciences (SPSS 22.0). Categorical variables were examined by the chi-square test and Fisher's exact test. Differences in continuous variables between the groups were tested by using Kruskal-Wallis test and the Mann-Whitney $U$ test; p values less than 0.05 were considered statistically significant.

\section{Results}

\subsection{Demographic data}

A total of 70 children (39 males and 31 females) with medically refractory TLE underwent resective surgery. Clinical features and preoperative evaluation of patients are summarized in Table 1 . The median age at seizure onset was 41.5 months ( 3 days-132 months). Forty-five patients (64.3\%) were younger than 12 years old, and 25 (35.7\%) were 12 years of age or older. The median duration of epilepsy until surgery was 71.6 months (1-194 months). The median age at the time of surgery was 127 months (10-238 months). Male gender was significantly higher in HS type 1 compared with other subgroups (p: 0.02).

\subsection{Seizures and antiepileptic drugs}

The most common seizure type was focal seizures (58 patients, 82.9\%). Twelve patients (17.1\%) had focal seizures evolving to bilateral tonic-clonic seizures.

Twenty-seven (38.6\%) patients had daily, 21(30\%) patients had weekly, and 22 (31.4\%) patients had monthly seizures. History of febrile seizures was noted in 25 patients (35.7\%); 13 patients (18.6\%) had history of admission for status epilepticus. History of febrile seizure was significantly higher in HS type 1 compared with other subgroups ( $\mathrm{p}$ : 0.012) (Table 1). 
Table 1

Demographic data and presurgical variables of all patients according to pathology.

\begin{tabular}{|c|c|c|c|c|}
\hline \multirow[t]{2}{*}{ Variables } & \multicolumn{4}{|c|}{ HS subtypes } \\
\hline & $\begin{array}{l}\text { HS type } 1 \\
\text { N: } 38(\%)\end{array}$ & $\begin{array}{l}\text { HS type } 2^{\mathrm{a}} \\
\mathrm{N}: 2(\%)\end{array}$ & $\begin{array}{l}\text { HS type } 3 \\
\mathrm{~N}: 21(\%)\end{array}$ & $\begin{array}{l}\text { No HS } \\
\text { N: } 9(\%)\end{array}$ \\
\hline \multicolumn{5}{|l|}{ Gender $^{\mathrm{b}, *}$} \\
\hline Male & $28(73.7)$ & $1(50)$ & $8(38)$ & $2(22.2)$ \\
\hline Female & $10(26.3)$ & $1(50)$ & $13(62)$ & $7(77.8)$ \\
\hline $\begin{array}{l}\text { Age at seizure onset/months } \\
\text { (median, range) }\end{array}$ & $\begin{array}{l}48 \\
(0.7-132)\end{array}$ & $\begin{array}{l}39,05 \\
(0,1-78)\end{array}$ & $\begin{array}{l}18 \\
(0.5-120)\end{array}$ & $\begin{array}{l}48 \\
(6-130)\end{array}$ \\
\hline Febrile seizure $^{\mathrm{b}, * *}$ & $19(50,0)$ & $1(50)$ & $3(14,2)$ & $2(22.2)$ \\
\hline Status epilepticus & $8(21,1)$ & $1(50)$ & $4(19)$ & $0(0)$ \\
\hline \multicolumn{5}{|l|}{ Seizure frequency } \\
\hline Daily & $14(36.8)$ & $1(50)$ & $10(47.6)$ & $2(22.2)$ \\
\hline Weekly & $11(28.9)$ & $1(50)$ & $6(28.5)$ & $3(33.3)$ \\
\hline Monthly & $13(34.2)$ & & $5(23.8)$ & $4(44.4)$ \\
\hline \multicolumn{5}{|l|}{ Seizure type } \\
\hline Focal & $31(81.5)$ & $1(50)$ & $17(81)$ & $9(100)$ \\
\hline Focal to bilateral tonic-clonic & $7(18.5)$ & $1(50)$ & $4(13)$ & \\
\hline \multicolumn{5}{|l|}{ Number of AED } \\
\hline 1 & $6(15.8)$ & & $5(23.8)$ & $3(33.3)$ \\
\hline $2-3$ & $31(81.6)$ & $1(50)$ & $14(66.6)$ & $6(66.7)$ \\
\hline$\geq 4$ & $1(2.6)$ & $1(50)$ & $2(9.5)$ & $0(.0)$ \\
\hline \multicolumn{5}{|l|}{ Preoperative EEG } \\
\hline Interictal focal discharges & $24(63.2)$ & $1(50)$ & $17(81)$ & $7(77.8)$ \\
\hline Ictal recording & $32(84.2)$ & $2(100)$ & 19(90.4) & $9(100)$ \\
\hline Focal/regional & $28(87.5)$ & $2(100)$ & $14(73.7)$ & $8(88.9)$ \\
\hline Lateralizing & $1(3.1)$ & & $1(5.2)$ & \\
\hline Nonlocalizing/nonlateralizing & $3(9.4)$ & & $4(21)$ & $1(11.1)$ \\
\hline \multicolumn{5}{|l|}{ Preoperative IQ } \\
\hline Not available & $7(18.4)$ & & $5(23.8)$ & \\
\hline Normal & $11(28.9)$ & $1(50)$ & $6(28.5)$ & $4(44.4)$ \\
\hline Borderline & $5(13.2)$ & & $2(9.5)$ & $1(11.1)$ \\
\hline Mild ID & $7(18.4)$ & & $7(33.3)$ & $4(44.4)$ \\
\hline Moderate ID & $8(21.1)$ & $1(50)$ & $1(4.8)$ & \\
\hline
\end{tabular}

HS: hippocampal sclerosis. AED: antiepileptic drug EEG: electroencephalogram IQ: intelligence quotient ID: intellectual disability.

a HS type 2 was not included in statistical analysis because of the small number of patients.

b Chi-square test for categorical variables.

* p: 0.02 .

** p: 0.012 .

All patients had been on two or more antiepileptic drugs (AEDs) preoperatively. At the time of surgery, 14 of 70 patients (20\%) were on one AED; 52 patients ( $74.3 \%$ ) were on 2 or 3 AEDs; 4 patients (5.7\%) were on more than 3 AEDs.

\subsection{Underlying etiologies and possible risk factors}

Underlying etiologies and possible risk factors were evaluated and classified via preoperative medical records, clinical, and neuroimaging data and were correlated with eventual pathological data. Twenty-five patients (35.7\%) had LEAT, 21 (30\%) had history of prolonged/recurrent febrile seizures, and 14 (20\%) had brain injury due to a variety of causes such as the following: head trauma (n: 4), central nervous system infection (n: 4), intracranial hemorrhage (n: 3); metabolic disorder (primary hypomagnesemia), acute lymphoblastic leukemia, and neonatal insult were diagnosed each in one patient. Two patients $(2.8 \%)$ had Rasmussen encephalitis. One patient had FCD IIb (1.4\%). The underlying etiology could not be clarified in 7 patients (10\%).

\subsection{Electroencephalogram and neuroimaging results}

Interictal EEG showed focal/regional abnormalities in forty-nine (70\%) patients; generalized or bilateral epileptiform discharges were not detected.
Ictal recordings could be obtained in 62 patients (88.6\%); 52 (83.9\%) had focal temporal or regional ictal onset ipsilateral with the resection side. Two patients (3.2\%) had ipsilateral ictal onset with the resection side but showed no localizing features on EEG. Ictal EEG failed to show localizing or lateralizing findings in 8 patients (12.9\%).

All patients revealed abnormal findings on presurgical brain MRI; 36 (51.4\%) patients had HS, 13 (18.6\%) patients had LEAT, 13 (18.6\%) patients had LEAT and involvement of hippocampi, 3 (4.2\%) patients had severe temporal atrophy, 3 (4.2\%) patients had suspected FCD, and 2 (2.9\%) patients had encephalomalacia. In sixty-five patients (92.9\%), seizure semiology and/or EEG findings were convergent with the lesion on MRI. Six patients (8.6\%) underwent functional MRI, 6 patients (8.6\%) had FDG-PET/CT; 7 patients (10\%) had interictal, and 3 (4.3\%) had ictal SPECT evaluation.

\subsection{Neuropsychological evaluation}

Presurgical neuropsychological evaluation was available for 58 patients; 22 patients (37.9\%) had normal intelligence quotient (IQ), 8 (13.8\%) had border line IQ 18 (31\%) had mild intellectual disability (ID), and 10 (17.2\%) had moderate ID.

\subsection{Surgical technique}

The resections were performed on the left in 35 patients (50\%) and on the right in 35 patients (50\%) (Table 2). Six (8.6\%) patients underwent second resection on the same temporal lobe owing to

Table 2

Etiology, surgical, and postsurgical variables of all patients according to pathology.

\begin{tabular}{|c|c|c|c|c|}
\hline \multirow[t]{2}{*}{ Variables } & \multicolumn{4}{|c|}{ HS subtypes } \\
\hline & $\begin{array}{l}\text { HS type } 1 \\
\mathrm{~N}: 38(\%)\end{array}$ & $\begin{array}{l}\text { HS type } 2^{\mathrm{a}} \\
\mathrm{N}: 2(\%)\end{array}$ & $\begin{array}{l}\text { HS type } 3 \\
\mathrm{~N}: 21(\%)\end{array}$ & $\begin{array}{l}\text { No HS } \\
\text { N: } 9(\%)\end{array}$ \\
\hline $\begin{array}{l}\text { Duration of epilepsy }{ }^{\mathrm{b}, *} \\
\text { (months) } \\
\text { (median, range) }\end{array}$ & $\begin{array}{l}80.5 \\
(15-194)\end{array}$ & $\begin{array}{l}114.5 \\
(108-120)\end{array}$ & $\begin{array}{l}41 \\
(1-129)\end{array}$ & $48(4-95)$ \\
\hline \multicolumn{5}{|l|}{ Etiology $\mathrm{c}^{\mathrm{c}, * *}$} \\
\hline LEAT & $3(7.9)$ & & $16(76.2)$ & $6(66.7)$ \\
\hline DNT & 1 & & 9 & 3 \\
\hline GG & & & 3 & 2 \\
\hline GNTu & 2 & & 3 & 1 \\
\hline LGA & & & 1 & \\
\hline Other & $17(44.7)$ & $1(50)$ & $4(19.1)$ & $2(22.2)$ \\
\hline $\begin{array}{l}\text { Prolonged/recurrent febrile } \\
\text { seizures }\end{array}$ & $18(47.4)$ & $1(50)$ & $1(4.7)$ & $1(11.1)$ \\
\hline $\begin{array}{l}\text { Age at surgery }{ }^{\mathrm{b}, * * *} \text { (months) } \\
\text { (median, range) }\end{array}$ & $\begin{array}{l}147 \\
(19-238)\end{array}$ & $\begin{array}{l}153.5 \\
(121-186)\end{array}$ & $\begin{array}{l}100 \\
(10-161)\end{array}$ & $\begin{array}{l}92 \\
(41-183)\end{array}$ \\
\hline \multicolumn{5}{|l|}{ Resection side } \\
\hline Right & $21(55.3)$ & & $10(47.6)$ & $4(44.4)$ \\
\hline Left & $17(44.7)$ & $2(100)$ & $11(52.4)$ & $5(55.6)$ \\
\hline \multicolumn{5}{|l|}{ Dual pathology } \\
\hline No & $35(92.1)$ & $1(50)$ & $2(9.5)$ & $9(100)$ \\
\hline Yes & $3(7.9)$ & $1(50)$ & $19(90.5)$ & 0 \\
\hline \multicolumn{5}{|l|}{ Postoperative EEG } \\
\hline Not available & $4(10.5)$ & & $2(9.5)$ & \\
\hline Available & $34(89.5)$ & $2(100)$ & $19(90.5)$ & $9(100)$ \\
\hline \multicolumn{5}{|l|}{$\begin{array}{l}\text { Interictal focal discharges on } \\
\text { EEG }\end{array}$} \\
\hline No & $29(85.3)$ & $1(50)$ & $13(68.4)$ & $8(88.9)$ \\
\hline Yes & $5(14.7)$ & $1(50)$ & $6(31.6)$ & $1(11.1)$ \\
\hline
\end{tabular}

HS: hippocampal sclerosis EEG: electroencephalogram.

LEAT: low-grade epilepsy-associated tumor; DNT: dysembryoplastic neuroepithelial tumor; GG: ganglioglioma; GNTu: glioneuronal tumor uncertain type; LGA: low grade astrocytoma.

${ }^{\text {a }}$ HS type 2 patients were not included in statistical analysis because of the small number of patients.

b Kruskal-Wallis test (Mann-Whitney U test for pairwise differences) for nonparametric data.

c Chi-square test for categorical variables.

* p: 0.031 .

** p: $<0.001$.

*** p: 0.007 . 
persistence of seizures, with a median duration of 31.5 months apart (8-87 months).

\subsection{Histopathologic findings and outcome}

Neuropathological evaluations revealed HS type 1 in 38 (54.3\%), HS type 2 in 2 (2.8\%), HS type 3 in 21 patients (30\%), and no HS in 9 patients (12.9\%) (Fig. 1). Two of HS type 1 cases were associated with architectural abnormalities in the temporal lobe and were referred as FCD Type IIIa. Patients with HS type 1 had statistically significant longer duration of epilepsy and an older age at the time of surgery; patients with HS type 3 and no HS had shorter duration of epilepsy and lower age at the time of surgery (p: 0.031, p: 0.007) (Table 2). Prolonged/recurrent febrile seizures were most frequent in HS type 1 group (18/38 patients, $47.4 \%)$.

Low-grade epilepsy-associated brain tumors were the leading diagnosis in patients with HS type 3 (16/21 patients, 76.2\%); LEAT were also common in no HS group $(6 / 9,66.7 \%)$. Underlying etiologies and possible risk factors were significantly different in HS subgroups ( $\mathrm{p}<0.001)$.

Only two patients were classified as ILAE type 2 ; one with intracranial hemorrhage, and the other with prolonged/recurrent febrile seizures. Hippocampal sclerosis type 2 subgroup was not included in statistical analysis because of the small number of patients.

Of 70 patients, 23 patients (32.9\%) had additional histopathologically confirmed principal ipsilateral temporal lobe pathology besides HS and were accepted as 'dual pathology'. The major HS subtype in patients with dual pathology was HS type 3 (19/23). Of these 19 patients with HS type 3, 16 had LEAT, 2 were diagnosed with Rasmussen encephalitis, and one patient had FCD IIb. A patient with glial scar formation showed HS type 2, and the other 3 patients with LEAT had HS type 1 (Table 3).

The distribution of HS types with respect to age revealed that in HS type 1 subgroup, 18 patients (47.4\%) were younger than 12 years, whereas 20 patients (52.6\%) were 12 years or older. Hippocampal sclerosis type 2 , which is represented by 2 patients, had one patient in each
Table 3

Distribution of patients with dual pathology $(n=23)$ across HS ILAE types.

\begin{tabular}{lllll}
\hline & HS type 1 & HS type 2 & HS type 3 & Total \\
\hline Second principal lesion & $3(13.1 \%)$ & $1(4.3 \%)$ & $19(82.6 \%)$ & $23(100 \%)$ \\
Glial scar & & 1 & & 1 \\
Rasmussen encephalitis & & & 2 & 2 \\
FCD type IIb & 3 & 1 & 1 \\
LEAT & 3 & 16 & 19 \\
\hline
\end{tabular}

HS: hippocampal sclerosis, FCD: focal cortical dysplasia, LEAT: low-grade epilepsy-associated tumor.

age group. In HS type 3 subgroup, 19 patients (90.5\%) were younger than 12 years, and 2 patients (9.5\%) were 12 years or older; age distribution for patients with no HS was 7 (77.8\%) and 2 (22.2\%), respectively. Hippocampal sclerosis type 3 and no HS subgroups had significantly more patients who were younger than 12 years (p: 0.002 ).

Low-grade epilepsy-associated brain tumors were the most common etiology in patients under 12 years of age (92\% vs $8 \%$ ), while prolonged/recurrent febrile seizures were most common in patients 12 years and older (38.1\% vs 61.9\%) ( $\mathrm{p}<0.001)$.

Postoperative EEG was available in 64 patients (91.4\%); 51 (79.7\%) had no interictal epileptiform discharges, whereas 13 (20.3\%) had residual interictal epileptiform discharges (Table 2).

The median follow-up duration after surgery was 70 months (24211 months). At the first visit within 6 months following surgery, sixty of 70 patients (85.2\%) were classified as Engel class I, 7 (10\%) as Engel class II, 1 (1.4\%) as Engel class III, and 2 (2.9\%) as Engel class IV (Table 4).

At final visit, 52 patients (74.3\%) were classified as Engel class I, 51 of them (98.1\%) were seizure-free since surgery and were classified as Engel class IA, one patient (1.9\%) had few disabling seizures after surgery due to compliance failure, yet she has remained completely seizure-free for the last two years. Ten patients were classified as Engel class II (14.3\%), 4 patients as Engel class III (5.7\%), and 4 patients as Engel class IV (5.7\%). Twenty-two patients (31.4\%) were off AED
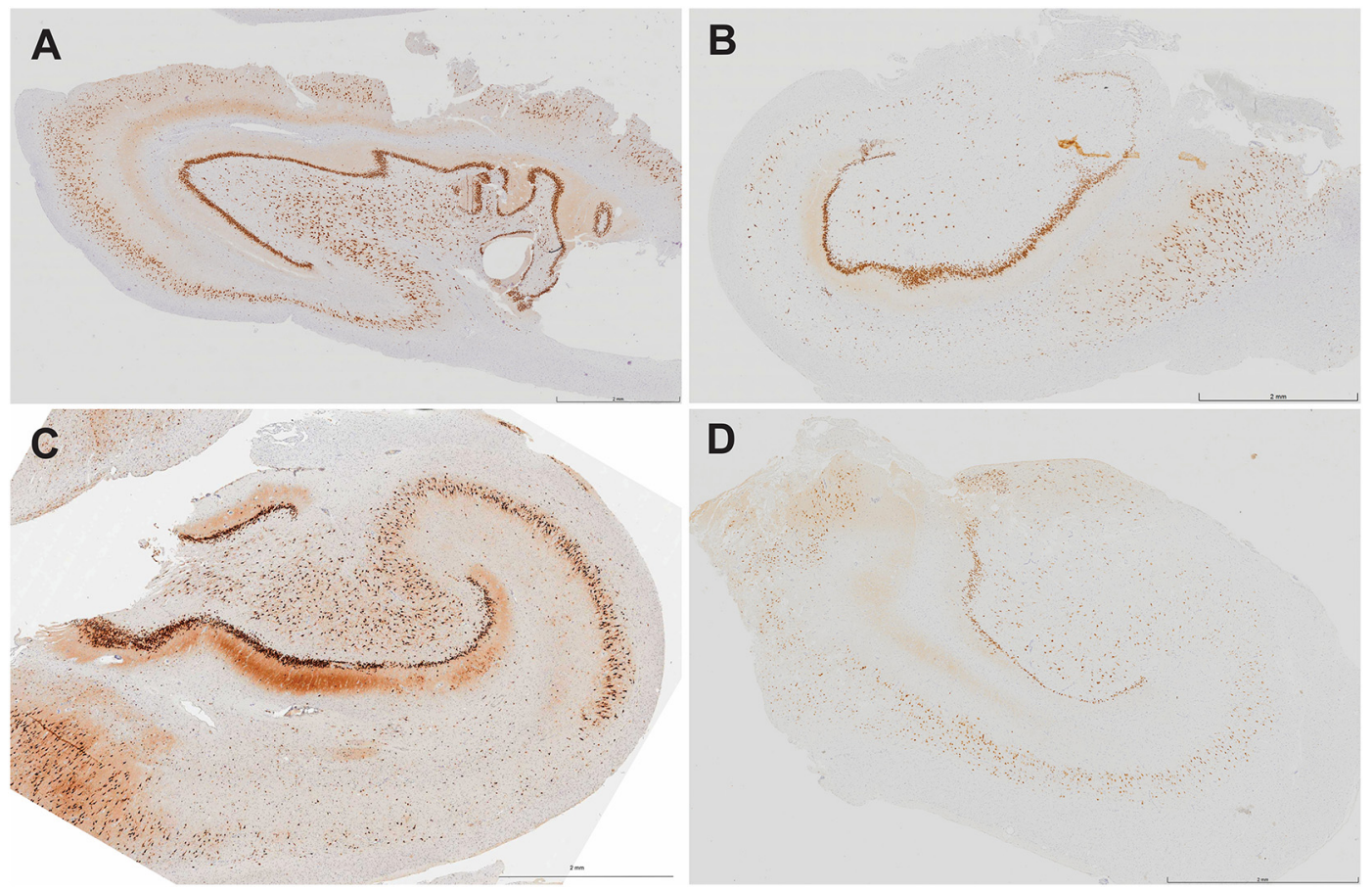

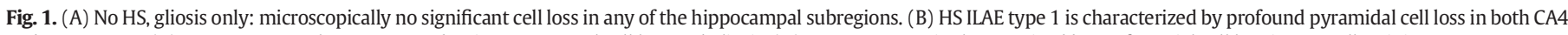

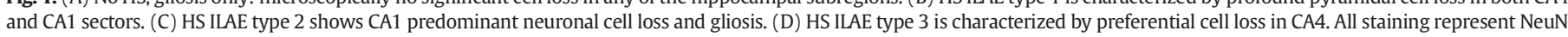
immunohistochemistry with hematoxylin counterstaining using $4-\mu \mathrm{m}$-thin paraffin embedded sections. Each photo consists of scale bar. 
Table 4

Postsurgical seizure outcome of all patients according to pathology.

\begin{tabular}{|c|c|c|c|c|}
\hline \multirow[t]{2}{*}{ Variables } & \multicolumn{4}{|c|}{ HS subtypes } \\
\hline & $\begin{array}{l}\text { HS type } 1 \\
\mathrm{~N}: 38(\%)\end{array}$ & $\begin{array}{l}\text { HS type } 2^{\mathrm{a}} \\
\mathrm{N}: 2(\%)\end{array}$ & $\begin{array}{l}\text { HS type } 3 \\
\mathrm{~N}: 21(\%)\end{array}$ & $\begin{array}{l}\text { No HS } \\
\text { N: } 9(\%)\end{array}$ \\
\hline \multicolumn{5}{|c|}{ Engel classification (within 6 months after surgery) } \\
\hline Class I & $34(89.5)$ & $2(100.0)$ & $16(76.1)$ & $8(88.9)$ \\
\hline Class II & $3(7.9)$ & & $4(19)$ & \\
\hline Class III & $1(2.6)$ & & & \\
\hline Class IV & & & $1(4.8)$ & $1(11.1)$ \\
\hline $\begin{array}{l}\text { Postoperative follow-up duration } \\
\text { (months) (median, range) }\end{array}$ & $\begin{array}{l}71.5 \\
(24-211)\end{array}$ & $\begin{array}{l}142 \\
(90-194)\end{array}$ & $\begin{array}{l}39 \\
(24-148)\end{array}$ & $\begin{array}{l}79 \\
(50-98)\end{array}$ \\
\hline \multicolumn{5}{|l|}{ Number of AED at final visit } \\
\hline No AED & $11(28.9)$ & & $3(14.2)$ & $6(66.7)$ \\
\hline 1 & $11(28.9)$ & $1(50.0)$ & $4(19)$ & \\
\hline $2-3$ & $13(34.2)$ & $1(50.0)$ & $14(66.7)$ & $3(33.3)$ \\
\hline$\geq 4$ & $3(7.9)$ & & & \\
\hline \multicolumn{5}{|l|}{ Engel classification (final visit) } \\
\hline Class I & $27(71.1)$ & $2(100)$ & $15(71.4)$ & $8(88.9)$ \\
\hline Class II & $9(23.7)$ & & $1(4.8)$ & \\
\hline Class III & $1(2.6)$ & & $3(14.3)$ & \\
\hline Class IV & $1(2.6)$ & & $2(9.5)$ & $1(11.1)$ \\
\hline \multicolumn{5}{|l|}{ Postoperative IQ } \\
\hline Not available & $15(39.5)$ & & $7(33.3)$ & \\
\hline Normal & $8(21.1)$ & & $3(14.2)$ & $6(66.7)$ \\
\hline Border line & $5(13.2)$ & & $3(14.2)$ & \\
\hline Mild ID & $6(15.8)$ & & $4(19)$ & $3(33.3)$ \\
\hline Moderate ID & $4(10.5)$ & $1(33.3)$ & $3(14.2)$ & \\
\hline Severe ID & & $1(33.3)$ & $1(4.7)$ & \\
\hline
\end{tabular}

HS: hippocampal sclerosis IQ: intelligence quotient AED: antiepileptic drug ID: intellectual disability.

${ }^{\text {a }}$ HS type 2 was not included in statistical analysis because of the small number of patients.

treatment; 13 patients (18.6\%) were using one AED, 32 patients ( $45.7 \%)$ were on 2 or 3 AEDs, and 3 patients (4.2\%) were on more than 3 AEDs (Table 4).

With respect to HS subtypes, seizure freedom was achieved in $27 / 38$ in HS type 1 (71.1\%), all 2 patients in HS type 2 (100\%), 15/21 patients in HS type 3 (71.4\%), and 8/9 patients in no HS group (88.9\%). No significant difference in seizure freedom was detected between subgroups with HS (Table 4). Seventeen of 23 (73.9\%) patients in the dual pathology group and 35 of 47 (74.7\%) patients in the HS alone group were seizure-free. There was no significant difference in seizure freedom between dual pathology and HS alone group. Also, seizure-free outcome showed no significant difference between patients younger than 12 years and patients who were 12 years and older, $71.1 \%$ vs $80 \%$, respectively (p: 0.415).

Postoperative neuropsychological evaluation was available for 48 patients; 17 (35.4\%) had normal IQ 8 (16.6\%) had borderline IQ; 13 (27\%) had mild ID, $8(16,6 \%)$ had moderate, and $2(4,1 \%)$ had severe ID.

Patients who achieved seizure freedom had significantly lower preoperative seizure frequency than patients who continued to have seizures (p: 0.04) and showed significantly lower focal interictal epileptiform discharges on postoperative EEG (p: 0.03). There were no significant differences between patients who achieved seizure freedom and patients who had postoperative seizures with respect to age at seizure onset, age at the time of surgery, duration of epilepsy until surgery, presurgical interictal/ictal EEG findings, and pathological subtypes of HS and underlying etiologies.

\section{Discussion}

Epilepsy surgery has proven efficacy in both adults and children with medically refractory epilepsy particularly in TLE. In adult series, seizurefree outcome in temporal resections ranges between 41 and 78\% [9-12]. In pediatric series, seizure outcome varies in a wide range [13]. In a systematic review with 1002 pediatric patients who underwent temporal resections, seizure-free outcome was 76\% [14], yet this may decline during long-term follow-up [15]. Reliable predictors of favorable postoperative seizure outcome have been a main concern in many studies. Preoperative and postoperative factors, such as seizure types and etiologies (lesional vs nonlesional), have been analyzed [14,15]. However, information on HS ILAE types and postsurgical seizure outcome is sparse and involve adult patients [16-18]. One study included both pediatric and adult patients [19]. To our knowledge, current study is the first report on HS ILAE types and the association of long-term seizure outcome, in exclusively children.

More than half of the patients are classified as HS type 1 in previous reports with mainly adult patients, HS type 2 is the second most common group, HS type 3 and no HS group are represented with few patients $[16-18,20]$. In accordance with adult series, HS type 1 is the most common group in our series ( $\mathrm{n}: 38,54.3 \%)$. However, unlike adult series, we found HS type 3 ( $\mathrm{n}: 21,30 \%$ ) as the second most common subtype. In our series, only two patients are classified as HS type 2 and nine patients as no HS group.

The difference in distribution of subgroups from adult data may be due to the heterogeneous etiologic profile in childhood TLE with HS. Mesial temporal sclerosis is the leading pathological substrate in adult TLE; on the other hand, developmental epileptogenic tumors are common in childhood $[5,17,21,22]$. Similar to the pediatric epilepsy surgery series, we had more patients with dual pathology than adult cases (23/ $70,32.8 \%$ ) [3,23]. In our study, majority of children with HS type 3 had dual pathology (19/21); LEAT was the most common substrate in patients with HS type $3(16 / 21)$. We conclude that the HS type 3 is remarkably more in children compared with adult series due to its cooccurrence with developmental epileptogenic lesions such as LEAT. Furthermore, when we analyzed our data with respect to age, the proportion of patients younger than 12 years were significantly more in HS type 3 and no HS subtypes, which could be due to close association with LEAT.

In our cohort, subgroups with HS showed no statistically significant difference regarding presurgical seizure frequency, seizure type, and history of status epilepticus. Tezer et al. reported history of status epilepticus and secondarily generalized tonic-clonic seizures being less frequent in HS type 1 in adults [18].

Prevalence of initial precipitating injury (IPI) and febrile seizures among HS subtypes differed between studies; IPI/febrile seizures either showed higher prevalence in HS type 2 or was not significantly different between HS subtypes in adults $[17,18,20]$. However, Na et al. reported higher incidence of IPI in HS type 1, though they did not specify the nature of IPI [19]. In our study, history of febrile seizures was significantly more frequent in HS type 1 compared with other subgroups. Similar findings in these two studies may be related to the presence of both pediatric and adult patients in the report by Na et al.

The relationship of HS subtypes and the duration of epilepsy differed between studies [17-19]. Our patients with HS type 1 had statistically significant longer duration of epilepsy and a greater age at the time of surgery compared with other subtypes, which may imply that these patients have clinically less severe epilepsy, in contrast with the widespread pathological findings. Deleo et al. found that febrile seizures do not have a role in the pathological type of HS, and they proposed that patients with HS type 1 may have a remote epileptogenic network responsible for the widespread disruption of hippocampal network, resulting in the postsurgical findings [17]. Our findings suggest that febrile seizures may have an influence on the pathological subtype of HS in childhood.

Adult patients with HS type 2 had significantly shorter duration of epilepsy suggesting that CA1 was a vulnerable subfield $[17,19]$. In our study, HS type 3 had statistically significant shorter duration of epilepsy and lower age at the time of surgery; however, this may not reflect the severity of epilepsy, and it could be due to frequent association of HS type 3 with LEAT. 
Major deviation of our series from the pathological subgroups seen in adult patients is the small size of HS type 2 group. Blumcke et al. reported that IPI could be a significant predictor of hippocampal pathology [24]. They found that patients with classical pattern showing severe cell loss in CA1 and moderate neuronal loss in all other subfields excluding CA2 had IPI at less than 3 years old; patients with severe neuronal loss restricted to sector CA1 had IPI at a later age (mean: 6 years), whereas patients with neuronal loss restricted to the hilar region and patients with normal appearing hippocampus had IPI beyond the age of 13. Age-dependent pathological disruption of hippocampal networks may explain the difference in distribution of subtypes in adult and pediatric HS. Hippocampal sclerosis type 2 was represented by merely two patients in our series, which may suggest that pathological changes in HS were age-dependent.

Atypical forms of HS (types 2 and 3 ) and no HS group were related with poorer postsurgical seizure-free outcomes in adults [25,26]. However, our results showed that pediatric patients with all three types of $\mathrm{HS}$ and no HS achieved favorable postsurgical seizure-free outcome in long-term follow-up. Postoperative seizure outcome showed no significant difference between HS subtypes in our study. Our patients with dual pathology and HS alone also had similar seizure outcome. A recent study of 384 patients with age at surgery between 15.4 and 67.9 years (mean: 36.8 years) showed no significant difference between HS subtypes in long-term seizure outcome similar to our findings [16]. On the other hand, postoperative seizure outcome in patients with LEAT is the best among disease categories in surgical pathology of epilepsy $[5,27]$. Therefore, postsurgical seizure outcome in patients with HS type 3 in our study may represent seizure outcome in patients with LEAT regardless of the presence or absence of HS and HS type.

One of the limitations of this study was inadequate data regarding cognitive outcome. Not all children had cognitive evaluation available for pre- and postoperative comparison. Also, evaluation of cognitive outcome was limited to IQ measurement and, therefore, lacked specific tests for learning and memory, as well as information regarding language dominance and correlation between laterality and outcome. Because of the retrospective nature of the study, the age of febrile seizures and the latency between febrile seizure and first afebrile seizure were not available. Neuropathologic specimens were reviewed only by one neuropathologist, which was another limitation of the study; and no molecular studies, such as BRAF mutation, were performed during review of the cases.

We present HS ILAE pathological subtypes in an exclusively pediatric series with long-term follow-up from a single tertiary center.

\section{Conclusion}

The present study shows that the leading pathological subgroup with HS in children is HS type 1, similar with adult series; however, HS type 3 emerges as the second most predominant group owing to the high incidence of dual pathology, predominantly LEAT in children. Further studies are required regarding clinicopathological features of isolated HS in pediatric cohort. History of febrile seizures was significantly more frequent in HS type 1 . Seizure-free outcome was favorable; however, our data were not adequate to comment on cognitive outcome. We conclude that the current classification of HS ILAE types highlights the difference of pathological substrates underlying childhood and adult patients with TLE and HS. As the current HS ILAE classification becomes more widely used, the proportion of HS types may be better defined in children with temporal resections and may help reveal the electroclinical correlates, surgical and cognitive outcome with respect to HS types.

\section{Disclosure}

A part of this study was presented as poster at the Annual Meeting of the American Epilepsy Society in 2015, in Philadelphia, Pennsylvania.

\section{Declaration of competing interest}

None of the authors has any conflict of interest to disclose.

\section{Acknowledgments}

The research was conducted at Hacettepe University Faculty of Medicine with no financial support.

We thank Dennis J. Dlugos, MD, MSCE, Section Head, Epilepsy Division of Neurology and Epilepsy at Children's Hospital of Philadelphia for reviewing the manuscript.

\section{References}

[1] Vadera S, Kshettry VR, Klaas P, Bingaman W. Seizure-free and neuropsychological outcomes after temporal lobectomy with amygdalohippocampectomy in pediatric patients with hippocampal sclerosis. J Neurosurg Pediatr. 2012;10: 103-7.

[2] Blumcke I. Neuropathology of focal epilepsies: a critical review. Epilepsy Behav 2009;15:34-9.

[3] Lee YJ, Kang HC, Bae SJ, Kim HD, Kim JT, Lee BI, et al. Comparison of temporal lobectomies of children and adults with intractable temporal lobe epilepsy. Childs Nerv Syst. 2010;26:177-83.

[4] Bocti C, Robitaille Y, Diadori P, Lortie A, Mercier C, Bouthillier A, et al. The pathological basis of temporal lobe epilepsy in childhood. Neurology. 2003;60: 191-5.

[5] Blumcke I, Spreafico R, Haaker G, Coras R, Kobow K, Bien CG, et al. Histopathological findings in brain tissue obtained during epilepsy surgery. N Engl J Med. 2017;377: 1648-56.

[6] Blumcke I, Thom M, Aronica E, Armstrong DD, Bartolomei F, Bernasconi A, et al. International consensus classification of hippocampal sclerosis in temporal lobe epilepsy: a Task Force report from the ILAE Commission on Diagnostic Methods. Epilepsia. 2013;54:1315-29.

[7] Engel JJ, Van Ness P, Rasmussen TB, Ojemann L. Outcome with respect to epileptic seizures. In: Engel JJ, editor. Surgical treatment of the epilepsies. New York: Raven Press; 1993. p. 609-21.

[8] Blumcke I, Thom M, Aronica E, Armstrong DD, Vinters HV, Palmini A, et al. The clinicopathologic spectrum of focal cortical dysplasias: a consensus classification proposed by an ad hoc Task Force of the ILAE Diagnostic Methods Commission. Epilepsia. 2011;52:158-74.

[9] Sylaja PN, Radhakrishnan K, Kesavadas C, Sarma PS. Seizure outcome after anterior temporal lobectomy and its predictors in patients with apparent temporal lobe epilepsy and normal MRI. Epilepsia. 2004;45:803-8.

[10] Engel Jr J, McDermott MP, Wiebe S, Langfitt JT, Stern JM, Dewar S, et al. Early surgical therapy for drug-resistant temporal lobe epilepsy: a randomized trial. Jama. 2012; 307:922-30.

[11] Schmidt D, Baumgartner C, Loscher W. The chance of cure following surgery for drug-resistant temporal lobe epilepsy. What do we know and do we need to revise our expectations? Epilepsy Res. 2004;60:187-201.

[12] Al-Kaylani M, Konrad P, Lazenby B, Blumenkopf B, Abou-Khalil B. Seizure freedom off antiepileptic drugs after temporal lobe epilepsy surgery. Seizure. 2007;16:95-8.

[13] Spencer S, Huh L. Outcomes of epilepsy surgery in adults and children. Lancet Neurol. 2008;7:525-37.

[14] Englot DJ, Rolston JD, Wang DD, Sun PP, Chang EF, Auguste KI. Seizure outcomes after temporal lobectomy in pediatric patients. J Neurosurg Pediatr. 2013;12:134-41.

[15] Benifla M, Rutka JT, Otsubo H, Lamberti-Pasculli M, Elliott I, Sell E, et al. Long-term seizure and social outcomes following temporal lobe surgery for intractable epilepsy during childhood. Epilepsy Res. 2008;82:133-8.

[16] Mathon B, Bielle F, Samson S, Plaisant O, Dupont S, Bertrand A, et al. Predictive factors of long-term outcomes of surgery for mesial temporal lobe epilepsy associated with hippocampal sclerosis. Epilepsia. 2017;58:1473-85.

[17] Deleo F, Garbelli R, Milesi G, Gozzo F, Bramerio M, Villani F, et al. Short- and longterm surgical outcomes of temporal lobe epilepsy associated with hippocampal sclerosis: relationships with neuropathology. Epilepsia. 2016;57(2):306-15.

[18] Tezer FI, Xasiyev F, Soylemezoglu F, Bilginer B, Oguz KK, Saygi S. Clinical and electrophysiological findings in mesial temporal lobe epilepsy with hippocampal sclerosis, based on the recent histopathological classifications. Epilepsy Res. 2016;127:50-4.

[19] Na M, Ge H, Shi C, Shen H, Wang Y, Pu S, et al. Long-term seizure outcome for international consensus classification of hippocampal sclerosis: a survival analysis. Seizure. 2015;25:141-6.

[20] Calderon-Garciduenas AL, Mathon B, Levy P, Bertrand A, Mokhtari K, Samson V, et al. New clinicopathological associations and histoprognostic markers in ILAE types of hippocampal sclerosis. Brain Pathol. 2018;28:644-55.

[21] Savitr Sastri BV, Arivazhagan A, Sinha S, Mahadevan A, Bharath RD, Saini J, et al. Clinico-pathological factors influencing surgical outcome in drug resistant epilepsy secondary to mesial temporal sclerosis. J Neurol Sci. 2014;340:183-90.

[22] Tellez-Zenteno JF, Hernandez-Ronquillo L. A review of the epidemiology of temporal lobe epilepsy. Epilepsy Res Treat. 2012;2012:630853.

[23] Li LM, Cendes F, Andermann F, Watson C, Fish DR, Cook MJ, et al. Surgical outcome in patients with epilepsy and dual pathology. Brain. 1999;122(5):799-805. 
[24] Blumcke I, Pauli E, Clusmann H, Schramm J, Becker A, Elger C, et al. A new clinicopathological classification system for mesial temporal sclerosis. Acta Neuropathol. 2007;113:235-44.

[25] Thom M, Liagkouras I, Elliot KJ, Martinian L, Harkness W, McEvoy A, et al. Reliability of patterns of hippocampal sclerosis as predictors of postsurgical outcome. Epilepsia. 2010;51:1801-8
[26] Thom M. Review: hippocampal sclerosis in epilepsy: a neuropathology review. Neuropathol Appl Neurobiol. 2014;40:520-43.

[27] Giulioni M, Marucci G, Pelliccia V, Gozzo F, Barba C, Didato G, et al. Epilepsy surgery of "low grade epilepsy associated neuroepithelial tumors": a retrospective nationwide Italian study. Epilepsia. 2017;58:1832-41. 